\title{
SCCL: FGFR1-Amplifikation mit stärkerem Zigarettenkonsum und einer schlechteren Prognose assoziiert
}

\begin{abstract}
Beim Plattenepithelkarzinom der Lunge (SCCL) ist der Nutzen der Chemotherapie sehr begrenzt, sodass intensiv nach zielgerichteten Therapieansätzen gesucht wird. Offenbar kommt der Amplifikation des Fibroblast Growth Factor Receptor-1-Gens (FGFR1) eine wichtige Rolle in der Onkogenese zu.
\end{abstract}

$\mathrm{H}$ ye Ryun Kim und Kollegen untersuchten bei 262 Patienten mit SCCL, die zwischen 1998-2009 operiert worden waren, den FGFR1-Status mithilfe der Fluoreszenz-in-situ-Hybridisierung (FISH). Von einer FGFR1-Amplifizierung (FGFR1 amp+) wurde gesprochen, wenn im Tumor neun oder mehr Genkopien vorlagen. $13 \%$ der Patienten wiesen eine Amplifizierung auf. Sie hatte ein signifikant kürzeres krankheitsfreies Überleben (DFS; 26,9 vs. 94,6 Monate; $p$ $<0,001)$ und ein kürzeres Gesamtüberleben (OS; 51,2 vs. 115,0 Monate; $\mathrm{p}=$ $0,002)$ zur Folge, das auch in der multivariaten Analyse bestätigt wurde (adjus- tiert für Geschlecht, Raucherstatus, pathologisches Tumorstadium und adjuvante Chemotherapie). Die adjustierten Hazard Ratios (AHR) betrugen für das DFS 2,24 (95\%-Konfidenzintervall [95\%-KI] 1,45-3,45; p < 0,001) und für das OS 1,83 (95\%-KI 1,15-2,89; p = 0,01). Aktive Raucher hatten signifikant öfter eine FGFR1 amp+ als ehemalige oder Niemals-Raucher (28,9 vs. 2,5 vs. $0 \%$; $\left.\mathrm{p}_{\text {trend }}<0,001\right)$ und die Häufigkeit stieg mit den Packungsjahren $\left(\mathrm{p}_{\text {trend }}=0,002\right)$.

In früheren Studien wurde eine vermehrte FGFR1-Amplifizierung auch bei Weißen und Japanern nachgewiesen. In weiteren prospektiven Studien müssen diese Ergebnisse für unabhängige Kohorten speziell auch in westlichen Ländern noch bestätigt werden.

Fazit: Die FGFR1-Genamplifikation ist ein unabhängiger negativer prognostischer Faktor beim operierten SCCL. Sie ist dosisabhängig assoziiert mit Zigarettenrauchen, sodass hier eine onkogene Aberration vorliegen dürfte. FGFR1 amp+ könnte einen wichtigen Angriffspunkt für eine zielgerichtete SCCLTherapie darstellen. Mehrere selektive FGFR-Tyrosinkinaseinhibitoren befinden sich bereits in früher klinischer Entwicklung.

Brigitte Schalhorn

Kim HR et al. Fibroblast growth factor receptor 1 gene amplification is associated with poor survival and cigarette smoking dosage in patients with resected squamous cell lung cancer. J Clin Oncol. 2013;31(6):731-7.

\section{Versorgung am Lebensende: Bessere Dokumentation der Patientenwünsche}

Ob ein Patient am Lebensende reanimiert werden will oder nicht, sollte man mit ihm beizeiten diskutieren. Eine Erinnerungs-E-Mail könnte dafür sorgen, dass der Patientenwunsch öfter als bisher erfragt und dokumentiert wird.

— ine qualitativ hochwertige Krebsthe- rapie sollte die Wünsche des Patienten hinsichtlich der medizinischen Versorgung am Lebensende und einer eventuellen Wiederbelebung berücksichtigen. Idealerweise spricht der Onkologe mit ihm darüber sprechen, lange bevor es zum lebensbedrohlichen Ereignis kommt. Außerdem sollte der Patientenwunsch dokumentiert werden, was jedoch selbst bei Patienten mit unheilbaren Erkrankungen nur selten geschieht.

Ziel der vorliegenden Studie war es, Onkologen durch eine E-Mail-Erinnerung dazu zu ermutigen, mit dem Patienten dieses Gespräch zu führen und seine Wünsche auch zu dokumentieren. 100 Patienten mit unheilbaren Lungenkarzinomen wurden 100 historischen Kontrollen - ebenfalls mit unheilbarem Lungenkarzinom - gegenübergestellt.
Um den optimalen Inhalt und Zeitpunkt der elektronischen Erinnerung zu bestimmen, wurden zunächst zwei Gruppen onkologisch tätiger Ärzte befragt. Danach wurde eine Nachricht entwickelt und per E-Mail versendet, mit der die Ärzte an das Gespräch zur Versorgung am Lebensende erinnert wurden.

Nach einem Jahr war bei $33,7 \%$ der Patienten, die an der Studie teilnahmen, das Gespräch in der Akte dokumentiert, dagegen nur bei 14,5\% der historischen Kontrollen $(\mathrm{p}=0,003)$. Bei den historischen Kontrollen erfolgte die Dokumentation häufiger erst während eines Krankenhausaufenthalts (2,0 vs. $13,0 \%$; $\mathrm{p}=$ $0,005)$. Auch die mittlere Zeit bis zur Dokumentation der Patientenwünsche bezüglich einer Reanimation war bei Studienteilnehmern signifikant kürzer $(8,6$ vs. 10,5 Monate; $\mathrm{p}=0,004$ ).

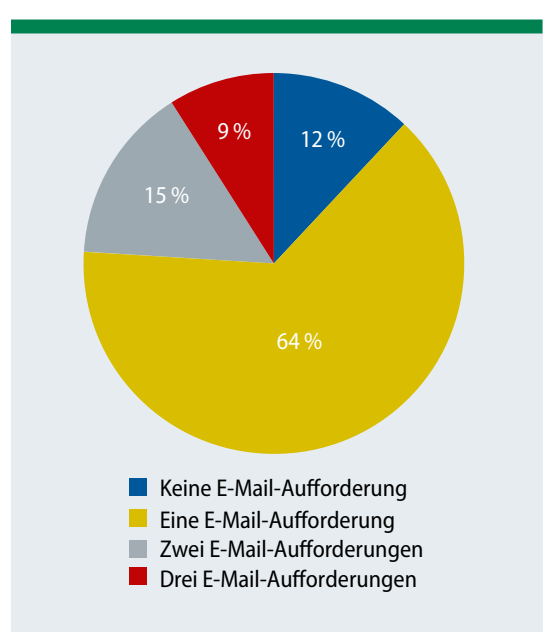

Abb.: Ein klarer Zusammenhang: E-MailErinnerungen fördern die Dokumentation des Patientenwunsches.

Fazit: Per E-Mail versendete Erinnerungen könnten die Dokumentation der Patientenwünsche hinsichtlich der Versorgung am Lebensende signifikant verbessern.

Judith Neumaier

Temel JS et al. Electronic prompt to improve outpatient code status documentation for patients with advanced lung cancer. J Clin Oncol. 2013;31(6):710-5. 